\title{
Elaboración de un simulador de trauma torácico a partir de un torso cadavérico utilizando tecnología de imágenes digitales e impresión 3D
}

\author{
Sebastián Spoerer ${ }^{1}$, Javier Vela ${ }^{2,3}$, Caterina Contreras ${ }^{2,3}$, Catalina Ortiz ${ }^{2,3}$, \\ Iván Caro ${ }^{1}$, Carlos Riquelme ${ }^{4}$, Francisco Garrido ${ }^{4}$, Óscar Inzunza ${ }^{5}$, \\ Juan Pablo Ramos ${ }^{3,6}$, Pablo Achurra ${ }^{2,3,6}$, Julián Varas ${ }^{2,3,6}$ y Nicolás Jarufe ${ }^{3}$
}

'Escuela de Diseño, Facultad de Arquitectura, Diseño y Estudios Urbanos, Pontificia Universidad Católica de Chile. Santiago, Chile. Departamento de Cirugía Digestiva, Facultad de Medicina, Pontificia Universidad Católica de Chile. Santiago, Chile.

${ }^{3}$ Centro de Simulación

y Cirugía Experimental Pontificia Universidad Católica

de Chile Santiago Chile. "Departamento de Radiología Facultad de Medicina, Pontificia Universidad Católica de Chile. Santiago, Chile.

5Departamento de Anatomía, Facultad de Medicina

Pontificia Universidad Católica de Chile. Santiago, Chile.

${ }^{6}$ Unidad de Trauma y Urgencias, Hospital Sótero de Río. Santiago, Chile.

Financiamiento: El presente trabajo ha sido financiado por CONICYT FONDECYT REGULAR

No 1171908: "Evaluación

de las competencias para

el manejo de trauma grave adquiridas con la formación

quirúrgica actual y utilidad del aprendizaje simulado para la adquisición de habilidades falentes"

Recibido 2020-06-19 y aceptado 2020-06-23

Correspondencia a Dr. Nicolás Jarufe njarufe@clinicalascondes.c
Elaboration of a thoracic trauma surgery simulation model from a cadaveric human torso incorporating digital image technology and 3D printing

Aim: To describe the design and creation of a high-fidelity thoracic trauma surgery simulation model incorporating 3D printing technology using a cadaveric human torso as a model. Materials and Method: This is a descriptive study that aims to illustrate the creation process of a thoracic trauma surgery simulation model throughout the incorporation of prototypes and dynamic iteration technologies. Results: A high-fidelity reusable thoracic trauma surgery simulation model was created from the digitalization of a cadaveric torso using a computed tomography scan. Throughout digital reconstruction tools, the subcutaneous, muscular, and skeletal structures were modeled from images obtained before and after an anterolateral thoracotomy. Using 3D printing and synthetic materials, a high-fidelity thoracic cavity was built so that perfused and ventilated porcine heart and lungs could be placed. A thoracotomy patch for the anterolateral thoracotomy was designed in a reusable and lowcost fashion. This simulation model is suitable for high fidelity training in the surgical management of cardiopulmonary traumatic injuries. Conclusion: The described methodology allowed the creation of a simulation model for training and assessment of surgical skills in thoracic trauma. The main components of the simulation model are made from reusable materials, broadening access to low-cost, high fidelity training.

Key words: simulation; thoracic surgery; medical education; 3-D Printing.

\section{Resumen}

Objetivo: Presentar la elaboración de un simulador de trauma torácico de alta fidelidad elaborado mediante modelamiento e impresión 3D a partir de un torso humano cadavérico. Materiales y Método: Estudio descriptivo del desarrollo de un simulador de trauma torácico utilizando metodología centrada en el prototipado y la iteración basada en testeos. Resultados: Se elaboró un simulador reutilizable mediante la digitalización de un torso cadavérico utilizando tomografía computada. Se realizó una reconstrucción digital del torso diseñando los planos subcutáneos, muscular y óseo en base a las imágenes del paciente pre y postoracotomía anterolateral. Utilizando impresión 3D y materiales sintéticos, se elaboró la caja torácica para luego instalar un corazón y pulmón porcino ventilado y perfundido. Los parches de la toracotomía son reemplazables y de bajo costo. En conjunto, este simulador permite el entrenamiento en manejo de lesiones traumáticas cardiacas y pulmonares de alta fidelidad. Conclusión: La metodología presentada permite la creación de un modelo para el entrenamiento y evaluación de habilidades quirúrgicas en trauma torácico. Los elementos principales del simulador son reutilizables y permiten mantener bajos los costos del entrenamiento.

Palabras clave: simulación; trauma torácico; educación; impresión 3D 


\section{Introducción}

El trauma representa la primera causa de muerte y discapacidad a nivel global ${ }^{1}$. A su vez, se estima que el $34,5 \%$ de estas defunciones podrían ser evitadas con mejoras en los sistemas de atención' 1 . Al analizar los pacientes hospitalizados por esta patología destaca que un $10 \%$ pertenece a trauma torácico, siendo este un marcador de mortalidad y severidad ${ }^{2,3}$. En el manejo de estos pacientes, la toracotomía de resucitación es un procedimiento capaz de salvar vidas, obteniendo tasas de sobrevida de $11,16 \%$ en heridas penetrantes torácicas, y hasta $31,1 \%$ en el caso de lesiones penetrantes cardiacas $^{4,5}$.

La evaluación y manejo quirúrgico del trauma corresponde a competencias del cirujano general según lo estipulado por la Sociedad Chilena de Cirujanos $^{6,7}$. Sin embargo, el contexto de la educación en cirugía está cambiando: la restricción de las horas laborales, mayores demandas de seguridad por parte de los pacientes y énfasis en el manejo conservador del trauma, han llevado a los residentes de cirugía a una menor exposición al trauma ${ }^{8-11}$. Este fenómeno también se ha dado en países como Estados Unidos y Canadá, donde el número de casos resueltos por residentes durante su periodo de formación ha disminuido progresivamente ${ }^{12,13}$. En nuestro país, según datos iniciales, aproximadamente un cuarto de los cirujanos recién egresados nunca realizó una toracotomía de urgencia durante su formación.

Por otra parte la simulación se ha consolidado como un complemento validado en el entrenamiento para la adquisición de habilidades quirúrgicas, siendo especialmente útil en procedimientos de baja ocurrencia, pero de alto riesgo ${ }^{14,15}$. La simulación ha demostrado transferir las habilidades adquiridas en el entramiento a la sala operatoria, logrando así acortar las curvas de aprendizaje mediante la práctica deliberada, donde el ejercicio repetitivo y progresivo de la técnica facilita el aprendizaje mediante el reconocimiento del error y su corrección ${ }^{16-18}$. Además, provee de un ambiente seguro tanto para el alumno como para el paciente ${ }^{15,19}$. En Chile existe gran experiencia en simulación para cirugía laparoscópica, sin embargo, la cirugía abierta y el trauma se ha desarrollado en mucho menor medida en parte debido a la menor disponibilidad de modelos de entrenamiento y a su alto costo.

De la mano de la ingeniería, la tecnología biomédica se ha desarrollado de forma exponencial en los últimos 20 años ${ }^{20,21}$. Dentro de estos avances destaca la alta definición de las imágenes médicas, en especial las de tomografía computada (TC). Por medio de la integración de las imágenes creadas por rayos X, la TC entrega una imagen de alta definición de la anatomía de un paciente, permitiendo crear modelos tridimensionales de los órganos y tejidos corporales ${ }^{22,23}$. Tras procesar estas imágenes en complejos sistemas computacionales se pueden modelar e imprimir en 3D utilizando impresoras especialmente diseñadas en base a resinas duras y blandas (de látex/siliconas). Estos modelos impresos tienen múltiples aplicaciones en medicina y en educación quirúrgica ${ }^{24-27}$.

El objetivo de este trabajo es presentar la metodología de elaboración de un simulador de trauma torácico de alta fidelidad mediante modelamiento e impresión 3D a partir de un torso humano cadavérico.

\section{Materiales y Método}

\section{Diseño del estudio}

Se realizó un estudio descriptivo del desarrollo de un simulador de trauma torácico utilizando metodología centrada en el prototipado y la iteración basada en testeos. Este trabajo forma parte de un proyecto FONDECYT (Proyecto N 11717908 - "Evaluación de las competencias para el manejo de trauma grave adquiridas con la formación quirúrgica actual y utilidad del aprendizaje simulado para la adquisición de competencias falentes"), como parte del desarrollo de herramientas que permitan evaluar y entrenar habilidades quirúrgicas en el manejo del trauma grave. Este estudio fue aprobado por el comité de ética de nuestra institución (Código Proyecto: 170318009).

\section{Desarrollo del modelo}

Se realizó un trabajo conjunto entre la Escuela de Diseño, el Departamento de Anatomía y el Centro de Simulación y Cirugía Experimental de la Pontificia Universidad Católica de Chile.

Se planteó el desarrollo de un simulador de bajo valor adquisitivo, bajo costo operativo, alta fidelidad anatómica, reutilizable y representativo del contexto local. Se siguió una metodología de trabajo Lean, que se basa en un proceso iterativo de desarrollo de un producto mínimo viable y la realización de mejoras consecutivas en base a la evaluación por parte de los usuarios finales ${ }^{28}$. A fin de privilegiar la mayor fidelidad anatómica posible y mantener una alta costo-efectividad se planteó la elaboración de un torso principal resistente y un parche desechable y reemplazable de pared torácica. Al interior del simulador se instalan tejidos de porcino obtenidos en mataderos y son perfundidos y/o ventilados para mayor realismo ${ }^{29}$. 


\section{ARTÍ́CULO ORIGINAL}

\section{Resultados}

En la Figura 1 se observa el modelo final con la toracotomía realizada y un corazón porcino perfundido en su interior. Se compone de un torso reutilizable, un parche de pared intercambiable y tejido animal porcino perfundido.

\section{Digitalización 3D de torso cadavérico}

Para la elaboración del torso se realizó el modelamiento tridimensional y digitalización de un torso cadavérico fresco utilizando tomografía computada y scanner 3D (Creaform 3D). Se utilizó tejido cadavérico por las altas dosis de radiación necesarias para obtener imágenes de alta definición y por permitir la realización de una toracotomía para estudiar la retracción exacta de los tejidos con la exposición quirúrgica. Tras la aprobación del comité de ética y bioseguridad, el cadáver fue seleccionado para asegurar la representatividad de la realidad local, seleccionándose un torso femenino de 34 años con IMC de $28 \mathrm{~kg} / \mathrm{m}^{2}$ y sin antecedentes quirúrgicos. Posterior a la tomografía basal, se realizó una toracotomía antero-lateral y se escaneó con un finochetto sintético a fin de evaluar la distorsión de la anatomía y de los distintos tejidos a la deformación (Figura 2). Luego, se reconstruyó la imagen 3D mediante el software Mimics $3 \mathrm{D}^{\circledR}$ el que permite, además, separar los di- ferentes tejidos en planos separados (Figura 2). Tras la reconstrucción digital se diseñaron los bordes del modelo, además, de las cavidades internas.

\section{Torso principal}

El torso principal se realizó inicialmente en madera para crear el molde para, posteriormente, construirlo en poliestireno de alto impacto (de $3 \mathrm{~mm}$ de grosor). Para el fresado en 3D en madera se utilizó tecnología de diseño guiado por computador (computer numerical control, CNC). Esta tecnología talla robóticamente el modelo en madera en base a las imágenes computacionales (Figura 3).

El torso se construyó con 2 segmentos: anterior y posterior. El segmento anterior proporciona los reparos anatómicos topográficos y cuenta con una fenestra en la región torácica lateral izquierda para ubicar el parche de pared costal usado para el entrenamiento de una toracotomía (Figura 1). El segmento posterior estaba divido en 2 espacios por un diafragma que además aporta estabilidad a la compresión abdominal. En el abdomen del modelo se montó un sistema recolector de sangre simulada para utilizar tejidos perfundidos. En los costados del modelo se ubicaron aperturas para la salida de conexiones a tubos para ventilar y perfundir los tejidos de animales utilizados dentro del modelo (Figura 4).

Figura 1. Resultado final de modelo de toracotomía. Se observa el torso final $(\mathbf{A})$; con una toracotomía y Finocchietto (B) y corazón/ pulmón porcino in situ (C). En (D) ilustra las fracturas costales por exceso de tracción ocasionado por el Finocchieto similar a una situación real.

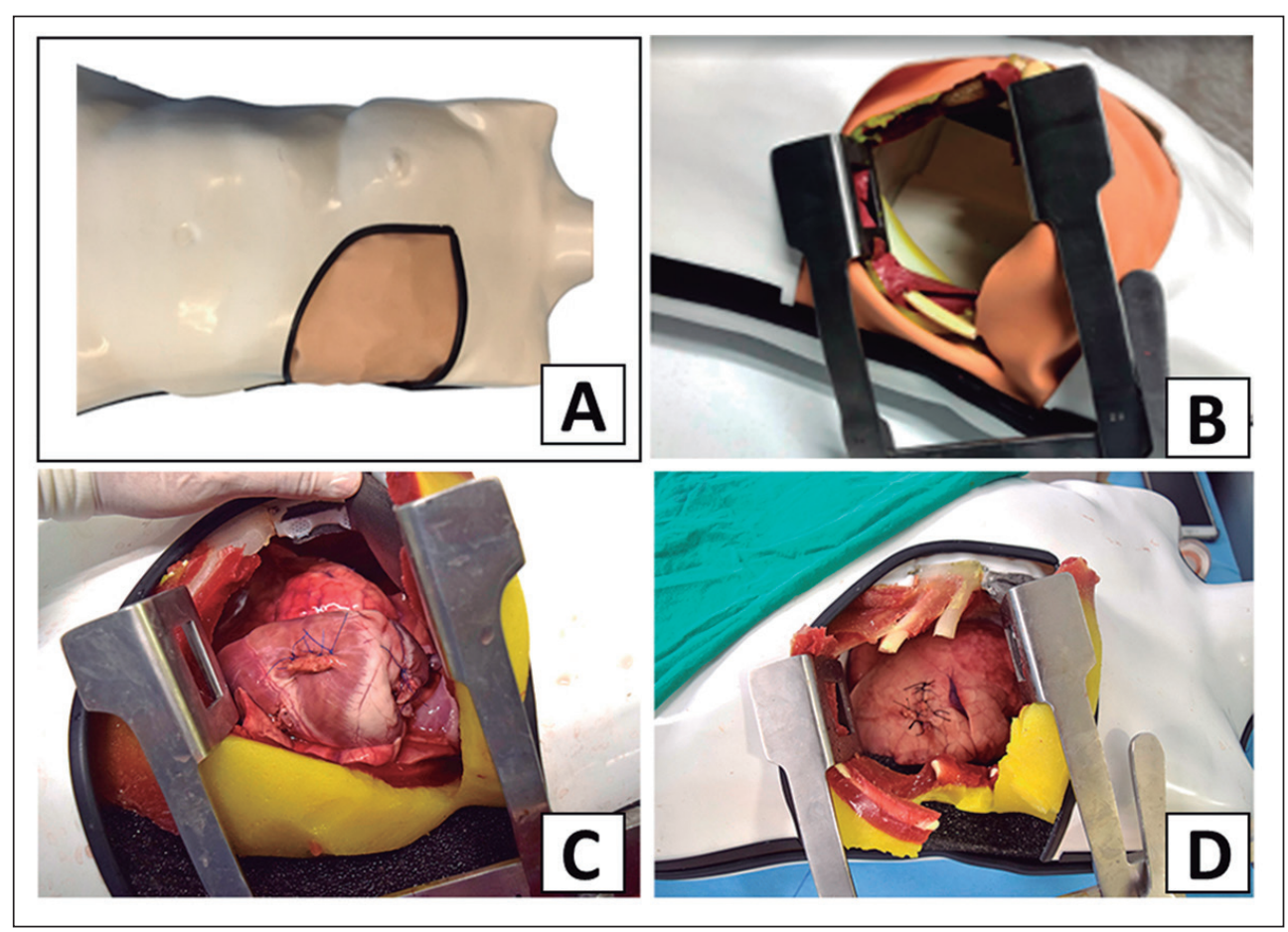



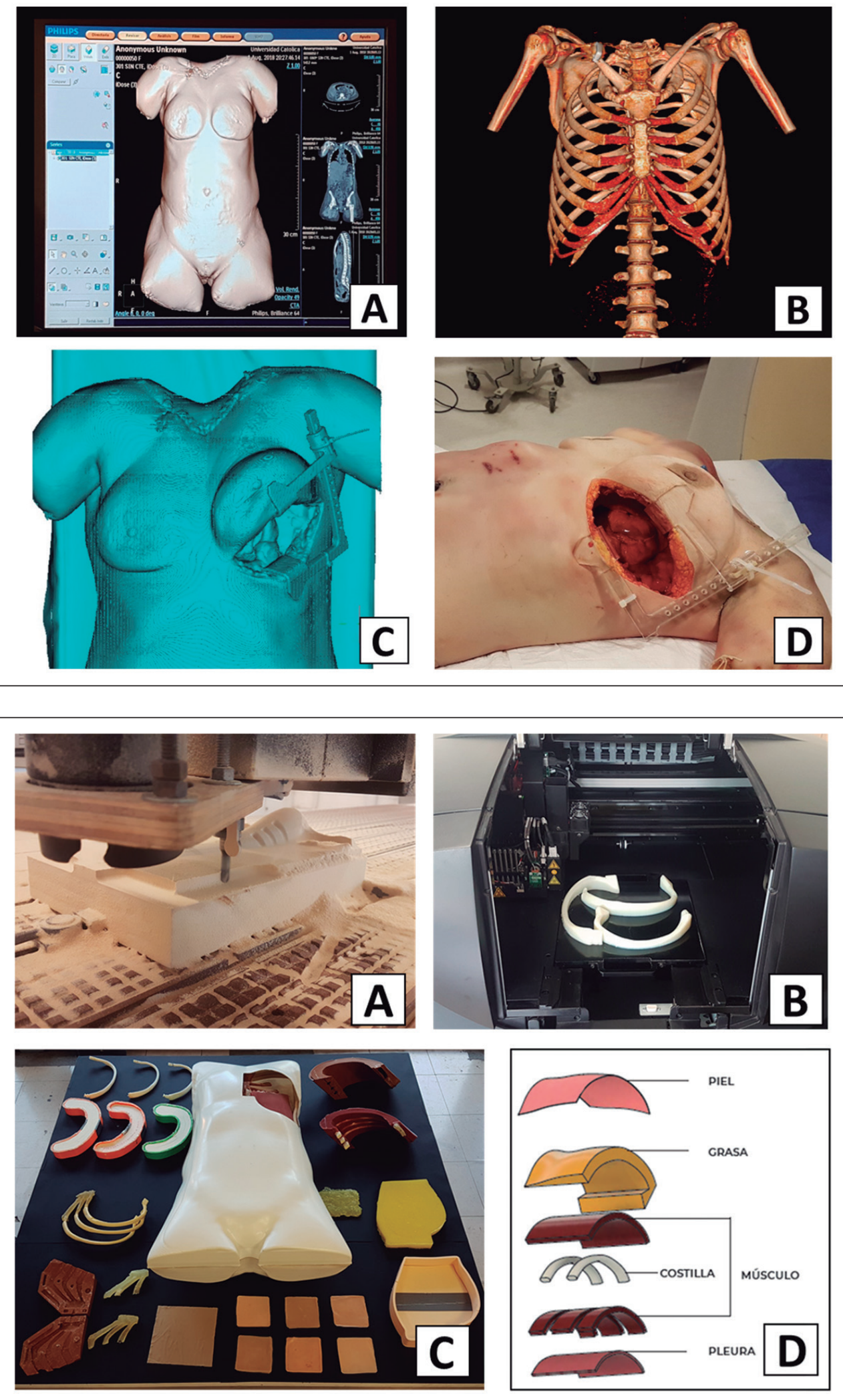

Figura 3. Distintos segmentos del simulador de trauma de tórax. En base a las imágenes y modelo 3D se realizó: Fresado robótico de molde en madera $(\mathbf{A})$, impresión 3D de componentes sólidos $(\mathbf{B})$, preparación y armado de componentes sintéticos (Cy $\mathbf{D})$. 


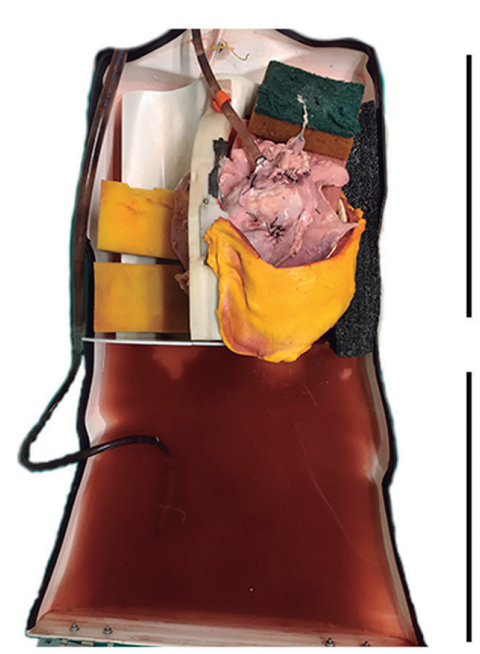

\section{Porción Torácica}

Segmento para Toracotomia y ubicación de corazón y pulmón porcino

Porción Abdominal

Reservorio de sangre artificial
Figura 4. Corazón perfundido y reservorio de sangre artificial. El sangrado producido por el modelo cae dentro del reservorio abdominal (piscina inferior). La sangre es extraída por la bomba desde el reservorio y nuevamente bombeada dentro del corazón en la porción torácica.

\section{Parche de pared costal desechable}

El parche de toracotomía se observa en la Figura 4D y cuenta con costillas, cartílagos y tejidos blandos cubiertos por una capa de piel sintética. A partir de las imágenes de TC se elaboraron moldes de costillas, esternón y cartílagos impresos en 3D en poliuretano con carga (impresora 3D Stratasys Objet 260 Connex y Zortrax M200). Las costillas fueron, posteriormente, incluidas en los tejidos blandos: músculo intercostal y tejido subcutáneo y cubiertas por una lámina de piel sintética. Estos tejidos fueron confeccionados con distintos materiales para representar con fidelidad la resistencia al corte y tracción de cada uno de ellos (silicona de caucho y ecoflex $\mathrm{TM} \mathrm{Gel}^{30}$ ). Las costillas permiten la fijación del parche de pared costal y se anclan por anterior al esternón y al segmento posterior. El costo de recambio de cada parche es de 30.000 pesos chilenos.

\section{Tejido cardiaco y pulmonar ex vivo}

Al interior del torso se ubicó un corazón porcino ex vivo con lesiones penetrantes en aurícula y ventrículo izquierdo y un pulmón porcino con lesiones transfixiantes a los que se acceden mediante la toracotomía antero-lateral realizada en el parche (Figura 1). El corazón es perfundido mediante una bomba roller que genera un flujo pulsátil de hasta $5 \mathrm{~L} / \mathrm{min}$ de sangre artificial. La inyección genera el efecto de latido cardiaco a 120-140 latidos por minuto. La sangre artificial cae dentro del modelo hacia el reservorio abdominal para luego recircular, permi- tiendo sangrado ilimitado. El pulmón es ventilado por un tubo orotraqueal utilizando un ambú manual o una máquina de anestesia. La ventilación permite evaluar la neumoestasia del pulmón posterior a la sutura.

\section{Discusión}

Se presenta un modelo de alta fidelidad para el entrenamiento y evaluación de habilidades quirúrgicas en trauma torácico. El simulador permite realizar una toracotomía anterolateral paso por paso seccionando piel, subcutáneo, músculo y pleura además de instalar correctamente un Finocchietto, para separación costal. Dentro del simulador se instalan tejidos animales que le dan mayor realismo a la reparación cardiaca y pulmonar. Su elaboración a partir de imágenes de un cadáver asegura la fidelidad de los reparos anatómicos. Además, la metodología empleada permite replicar a futuro el mismo proceso de elaboración para obtener modelos de otras poblaciones como, por ejemplo, pacientes obesos o pediátricos.

Existen múltiples modelos de simulación para trauma torácico en la literatura, sin embargo, la mayoría de estos son poco costo-efectivos para el entrenamiento ${ }^{31-33}$. Los modelos animales y cadavéricos si bien son de alta fidelidad, son de costo elevado y de difícil acceso para muchos centros; los modelos sintéticos son de menor costo, pero no logran una representación realista de los tejidos. En el modelo presentado se combina material sintético con tejidos animales preservando el realismo de los tejidos y el bajo costo y accesibilidad del material sintético.

La incorporación en el diseño de un parche de pared costal reemplazable y desechable es un elemento clave para asegurar bajos costos de operatividad. Permite que cada usuario del modelo practique la toracotomía sin necesidad de recambiar el torso completo. Además, la elaboración de moldes impresos en 3D, si bien son costosos en un inicio, permiten replicar a bajo costo los parches de pared costal (costillas, cartílagos, etc.).

Las limitaciones de este modelo incluyen su dependencia en tejidos animales para el manejo de lesiones pulmonares y cardiacas. La validación de su uso como modelo de evaluación y entrenamiento en cirugía está actualmente en investigación.

\section{Conclusión}

En este trabajo se presenta un modelo de entrenamiento de trauma de tórax de alta fidelidad rea- 
lizado en base a imágenes de un torso cadavérico, impresión 3D y tejidos animales. Los elementos principales del simulador son reutilizables y permiten mantener bajos los costos de entrenamiento.

\section{Agradecimientos}

Agradecemos a Microgeo por su ayuda en facilitar y orientarnos en el uso de impresoras 3D.

\section{Responsabilidades éticas}

Protección de personas y animales. Los autores declaran que para esta investigación no se han realizado experimentos en seres humanos ni en animales.

Confidencialidad de los datos. Los autores declaran que en este artículo no aparecen datos de pacientes.

Conflictos de interés: no hay.

\section{Bibliografía}

1. Mock C. WHO releases Guidelines for trauma quality improvement programmes. Inj Prev. 2009;15:359. doi: 10.1136/ ip.2009.024315.

2. Dennis BM, Bellister SA, Guillamondegui OD. Thoracic Trauma. Surg Clin North Am. 2017;97:1047-64. doi: 10.1016/j. suc.2017.06.009.

3. Majercik S, Pieracci FM. Chest Wall Trauma. Thorac Surg Clin. 2017;27:113121. doi: 10.1016/j.thorsurg.2017.01.004

4. Working Group, Ad Hoc Subcommittee on Outcomes, American College of Surgeons. Committee on Trauma. Practice management guidelines for emergency department thoracotomy. J Am Coll Surg 2001;193:303-9. doi: 10.1016/S10727515(01)00999-1.

5. Narvestad JK, Meskinfamfard M, Søreide $\mathrm{K}$. Emergency resuscitative thoracotomy performed in European civilian trauma patients with blunt or penetrating injuries: a systematic review. Eur J Trauma Emerg Surg. 2016;42:677-85. doi: 10.1007/ s00068-015-0559-z.

6. Bustamante ZM, Espinoza GR, Hepp KJ, Martínez CJ. Estándares de la formación del cirujano. Visión de la sociedad de cirujanos de Chile. Rev Chil Cir. 2015;67:102-8. doi: 10.4067/S071840262015000100017

7. Hepp JK, Csendes AJ, Ibáñez FC, Llanos OL, San Martín SR. Programa de la Especialidad Cirugía General. Definiciones y Propuestas de la Sociedad de Cirujanos de Chile Programa de La Especialidad Cirugía General. Rev Chil Cir. 2008;60:7985. http://dx.doi.org/10.4067/S071840262008000100017

8. Reznick RK, MacRae H. Teaching
Surgical Skills-Changes in the Wind. Cox M, Irby DM, eds. N Engl J Med. 2006;355:2664-9. doi: 10.1056/ NEJMra054785.

9. Klingensmith ME, Lewis FR. General surgery residency training issues. Adv Surg. 2013;47:251-70. doi: 10.1016/j. yasu.2013.05.001

10. Gunst M, O'Keeffe T, Hollett L, Hamill M, Gentilello LM, Frankel H, et al. Trauma Operative Skills in the Era of Nonoperative Management: The Trauma Exposure Course (TEC). J Trauma Inj Infect Crit Care 2009;67:1091-6. doi: 10.1097/ TA.0b013e3181bc77ba.

11. Mattar SG, Alseidi AA, Jones DB, Jeyarajah DH, Swanstrom LL, Aye RW, et al. General surgery residency inadequately prepares trainees for fellowship: Results of a survey of fellowship program directors. Annals of Surgery 2013;258:440-7. doi: 10.1097/SLA.0b013e3182a191ca

12. Strumwasser A, Grabo D, Inaba K, Matsushima K, Clark D, Benjamin E, et al. Is your graduating general surgery resident qualified to take trauma call? A 15-year appraisal of the changes in general surgery education for trauma. J Trauma Acute Care Surg. 2017;82:470-80. doi: 10.1097/ TA.0000000000001351.

13. Engels PT, Bradley NL, Ball CG. The current state of resident trauma training: Are we losing a generation? Can J Surg. 2018;61:153-4. doi: 10.1503/CJS.014417.

14. Cannon-Bowers JA, Bowers C, Procci K. Optimizing Learning in Surgical Simulations: Guidelines from the Science of Learning and Human Performance. Surg Clin North Am. 2010;90:583-603. doi: 10.1016/j.suc.2010.02.006

15. Sutherland LM, Middleton PF, Anthony A, Hamdorf J, Cregan P, Scott D, et al. Surgical Simulation. Ann Surg. 2006;243:291-300. doi: 10.1097/01. sla.0000200839.93965.26.

16. Varas J, Mejía R, Riquelme A, Maluenda F, Buckel E, Salinas J, et al. Significant transfer of surgical skills obtained with an advanced laparoscopic training program to a laparoscopic jejunojejunostomy in a live porcine model: feasibility of learning advanced laparoscopy in a general surgery residency. Surg Endosc. 2012;26:3486-94. doi: 10.1007/s00464-012-2391-4.

17. Choy I, Okrainec A. Simulation in Surgery: Perfecting the Practice. Surg Clin North Am. 2010;90:457-73. doi: 10.1016/j. suc.2010.02.011

18. McGaghie WC, Issenberg SB, Cohen ER, Barsuk JH, Wayne DB. Does Simulation-Based Medical Education With Deliberate Practice Yield Better Results Than Traditional Clinical Education? A Meta-Analytic Comparative Review of the Evidence. Acad Med. 2011;86:706-11. doi: 10.1097/ACM.0b013e318217e119.

19. Aggarwal R, Mytton OT, Derbrew M, Hananel D, Heydenburg M, Issenberg B, et al. Training and simulation for patient safety. Qual Saf Heal Care. 2010;19(Suppl 2):i34-i43. doi: 10.1136/qshc.2009.038562

20. Corvetto M, Pía Bravo M, Montaña R, Utili F, Escudero E, Boza C, et al. Simulación En Educación Médica: Una Sinopsis. Rev Med Chile 2013;141:709. http://dx.doi.org/10.4067/S00349887201300010001.

21. Depeursinge A, Foncubierta-Rodriguez A, Van De Ville D, Müller H. Threedimensional solid texture analysis in biomedical imaging: Review and opportunities. Med Image Anal. 2014;18:176-96. doi: 10.1016/j. media.2013.10.005. 


\section{ARTÍCULO ORIGINAL}

22. Kalender W. Computed Tomography: Fundamentals, System Technology, Image Quality, Applications. Wiley-VCH. 2011.

23. Mery D. Computer Vision for X-Ray Testing. Springer International Publishing 2015. doi: 10.1007/978-3-319-20747-6.

24. Tai BL, Rooney D, Stephenson F, Liao PS, Sagher O, Shih AJ, et al. Development of a 3D-printed external ventricular drain placement simulator: Technical note. J Neurosurg. 2015;123:1070-6. doi: 10.3171/2014.12.JNS141867

25. Malik HH, Darwood ARJ, Shaunak $\mathrm{S}$, et al. Three-dimensional printing in surgery: a review of current surgical applications. J Surg Res. 2015;199:512-22. doi: 10.1016/j.jss.2015.06.051.

26. Rankin TM, Giovinco NA, Cucher DJ, Watts G, Hurwitz B, Armstrong DG. Three-dimensional printing surgical instruments: Are we there yet? J Surg
Res. 2014;189:193-7. doi: 10.1016/j. jss.2014.02.020.

27. Potkonjak A, Hartman A. 3D printing of aortic heart valve tissue in patients suffering from cardiovascular disease. 2015.

28. Cohen RI. Lean Methodology in Health Care. Chest. 2018;154:1448-54. doi: 10.1016/j.chest.2018.06.005.

29. Ávila R, Achurra P, Tejos R, Perkis JR, Muñoz M, Salas P, et al. Validación de modelo de entrenamiento basado en simulación para adquirir habilidades en trauma complejo cardiopulmonar. Investig en Educ Médica. 2017;6:130. doi: 10.1016/j. riem.2017.01.125

30. Ecoflex ${ }^{\mathrm{TM}}$ GEL Product Information Smooth-On, Inc. https://www.smooth-on. com/products/ecoflex-gel/. Accessed June 172020.
31. Sánchez LD, Delapena J, Kelly SP, Ban K, Pini R, Perna AM. Procedure lab used to improve confidence in the performance of rarely performed procedures. Eur J Emerg Med. 2006;13:2931. http://www.ncbi.nlm.nih.gov/ pubmed/16374245. Accessed October 15, 2019.

32. Ferguson IM, Shareef MZ, Burns B, Reid C. A human cadaveric workshop: One solution to competence in the face of rarity. Emerg Med Australas. 2016;28:7524. doi: 10.1111/1742-6723.12601.

33. Bohnen JD, Demetri L, Fuentes E, Butler $\mathrm{K}$, Askari R, Anand RJ, et al. High-Fidelity Emergency Department Thoracotomy Simulator With Beating-Heart Technology and OSATS Tool Improves Trainee Confidence and Distinguishes Level of Skill. J Surg Educ. 2018;75:1357-66. doi: 10.1016/j.jsurg.2018.02.001. 